\title{
Guest editorial: understanding the pathogenesis and the evolving treatment paradigm for multiple myeloma in the era of novel agents
}

\author{
Masahiro Abe
}

Received: 25 September 2011/Revised: 26 September 2011/Accepted: 26 September 2011/Published online: 13 October 2011

(C) The Japanese Society of Hematology 2011

\section{Shifts in the therapeutic paradigm}

The introduction of thalidomide, lenalidomide, and bortezomib has significantly improved the response rates and survival outcome in patients with myeloma (MM), and been integrated into MM treatment [1-3]. These new agents target not only the intracellular mechanisms in MM cells but also the interaction between MM cells and the bone marrow microenvironment. Given the clinical benefit of these novel agents either alone or in combinatorial regimens, the treatment of $\mathrm{MM}$ is changing rapidly. A focus of clinical studies appears to be the evaluation and identification of novel combinations using these new agents to uniformly produce better outcomes in different clinical settings of patients with MM, and to further tailor treatment as appropriate to the clinical background as well as the biological characteristics of MM cells and their surrounding microenvironment in individual patients.

High-dose therapy with autologous hematopoietic stem cell transplantation (ASCT) is the standard of care for newly diagnosed MM patients eligible for ASCT. Introduction of new drugs in the induction setting have markedly improved response rates, leading to increased survival rates after ASCT. For newly diagnosed elderly patients, or those not eligible for ASCT, the introduction of novel agents has also changed the management of this disease. The combination of melphalan, prednisone, and thalidomide, and that of bortezomib, melphalan, and prednisone are regarded as the new standards of care for elderly patients [4]. However, no standard treatments have been established for relapsed/refractory MM, although it has

M. Abe $(\bowtie)$

University of Tokushima, Tokushima, Japan

e-mail: masabe@clin.med.tokushima-u.ac.jp been suggested that the implementation of treatment regimens based on proteasome inhibitors and/or immunomodulatory drugs (IMiDs) improves response rates and survival in patients with relapsed/refractory MM [5, 6]. Overall, recent data suggest that the upfront combination of individual proteasome inhibitors and IMiDs is highly effective, and new approaches with these novel agents are currently being studied to further improve the response rates and duration of response, including the implementation of induction therapies with three or more drugs in combination, and consolidation and maintenance therapies featuring long-term administration of novel agents.

\section{Risk-stratification by cytogenetic and molecular profiling}

MM is a molecularly heterogeneous disease with high genetic instability and epigenetic alterations. The development and progression of MM is a complex multi-step process, involving genetic changes in MM cells. Specific genetic alterations have been shown to be linked to patients' clinical presentation, response to treatment, and prognosis. MM can be divided largely into a hyperdiploid group with multiple trisomies, and a non-hyperdiploid group with immunoglobulin heavy chain $(\mathrm{IgH})$ translocations based on the findings of conventional cytogenetics and fluorescence in situ hybridization (FISH). These subtypes are associated with different clinicopathological features and outcomes. The latter group with $\mathrm{t}(4 ; 14)(\mathrm{p} 16 ; \mathrm{q} 32)$ or $\mathrm{t}(14 ; 16)(\mathrm{q} 32 ; \mathrm{q} 23)$ translocations represents a high-risk subtype with more aggressive clinical features and poorer prognosis. In contrast, the hyperdiploid group is generally considered a more indolent form with a better prognosis. Thus, chromosome ploidy status and $\operatorname{IgH}$ rearrangements are used to help 
subdivide patients with $\mathrm{MM}$ into different prognostic groups. During the course of the disease, MM cells eventually become more aggressive and acquire treatment resistance along with a number of secondary chromosomal aberrations, including c-Myc rearrangements, deletion of chromosome $17 \mathrm{p}$, and abnormalities of chromosome 1 (1p deletion and 1q amplification) [7, 8]. In the "Progress in Hematology" series in this issue of International Journal of Hematology, Knooper et al. report significant clinical impact of their risk-stratification with prognostic determinants, including chromosomal and cytogenetic aberrations in newly diagnosed $\mathrm{MM}$, and provide an outline of evidencebased management approaches focusing on risk-adapted therapy with their recommendations in transplant-eligible and ineligible patients from the induction to maintenance settings. Of note, the poor prognostic implications of $t(4 ; 14)$ have been overcome with bortezomib-based regimens; and thus patients with $t(4 ; 14)$ are now classified in the intermediate rather than high-risk category in the updated version of Mayo Clinic-proposed mSMART risk classification. However, deletion of chromosome $17 \mathrm{p}$ is consistently associated with poor prognosis, even on extensive treatment with new agents. Gene expression profiling (GEP), array comparative genomic hybridization (aCGH), and single-nucleotide polymorphism (SNP) arrays have made it possible to characterize patterns of genetic aberration and alteration in MM cells in a more sophisticated manner [9-11]. In this review series, Johnson et al. provide a comprehensive and informative review on the novel classification of MM based on GEP analyses and highlight the "GEP70" signature for defining the emerging molecular genetic subgroups closely associated with unique clinicopathological features and the treatment outcomes.

\section{Bone marrow microenvironment suited for MM growth (MM niche)}

The fact that MM cells grow and expand almost exclusively in the bone marrow suggests the importance of the bone marrow microenvironment in supporting MM cell growth and survival. In addition to intrinsic genetic changes in MM cells, the progression of $\mathrm{MM}$ is facilitated by the bone marrow microenvironment skewed by MM cells, which underlies the unique pathophysiology of MM [12-14]. MM cells enhance osteoclastogenesis and stimulate angiogenesis in concert with bone marrow stromal cells and osteoclasts, whereas they suppress osteoblastic differentiation from bone marrow stromal cells, leading to devastating bone destruction and the rapid loss of bone $[15,16]$. Importantly, osteoclasts, vascular endothelial cells, and bone marrow stromal cells with defective osteoblastic differentiation create a cellular microenvironment suitable for MM growth and survival and confer drug resistance [12, 17], in many senses, an "MM niche" [15]. This review series provides an overview of the current understanding of the mechanisms underlying the mutual interactions between MM cells and local bone marrow cells leading to the vicious cycle between bone destruction and tumor expansion.

\section{From MM biology to new therapy}

MM remains incurable despite the recent implementation of novel anti-MM agents along with high-dose chemotherapies and immunotherapies, indicating the strong need for continued investigation of innovative strategies. Further elucidation of the biology underlying the disease progression and the molecular mechanisms of drug resistance in MM cells in the MM niche will provide us with new approaches that have a real impact on both MM bone disease and tumor progression [18]. In this series of reviews, Hideshima et al. succinctly describe the current status of the development and validation of novel targeted therapies, and introduce preclinical and clinical observations of various novels agents, including those targeting growth factors and cell surface molecules, intracellular signaling pathways, protein catabolism and unfolded protein response, and cell cycle regulators in MM cells.

Hematologists and oncologists are now gaining access to a wider variety of therapeutic options for MM tailored to the most appropriate and effective treatment according to their patients' characteristics. This series will provide updated information on MM biology and treatment and will surely prove to be of interest and merit for readers of the International Journal of Hematology.

\section{References}

1. Laubach JP, Schlossman RL, Mitsiades CS, Anderson KC, Richardson PG. Thalidomide, lenalidomide and bortezomib in the management of newly diagnosed multiple myeloma. Expert Rev Hematol. 2011;4:51-60

2. Ludwig H, Beksac M, Blade J, Boccadoro M, Cavenagh J, Cavo $\mathrm{M}$, et al. Current multiple myeloma treatment strategies with novel agents: a European perspective. Oncologist. 2010;15:6-25.

3. Palumbo A, Attal M, Roussel M. Shifts in the therapeutic paradigm for patients newly diagnosed with multiple myeloma: maintenance therapy and overall survival. Clin Cancer Res. 2011;17:1253-63.

4. Gay F, Palumbo A. Management of older patients with multiple myeloma. Blood Rev. 2011;25:65-73.

5. Stewart AK. Novel therapies for relapsed myeloma. Hematol Am Soc Hematol Educ Program. 2009;578-86.

6. Richardson PG, Laubach J, Mitsiades C, Schlossman RL, Doss D, Colson $\mathrm{K}$, et al. Tailoring treatment for multiple myeloma patients with relapsed and refractory disease. Oncology (Williston Park). 2010;24:22-9. 
7. Fonseca R, Bergsagel PL, Drach J, Shaughnessy J, Gutierrez N, Stewart AK, et al. International Myeloma Working Group molecular classification of multiple myeloma: spotlight review. Leukemia. 2009;23:2210-21.

8. Avet-Loiseau H, Soulier J, Fermand JP, Yakoub-Agha I, Attal M, Hulin C, et al. Impact of high-risk cytogenetics and prior therapy on outcomes in patients with advanced relapsed or refractory multiple myeloma treated with lenalidomide plus dexamethasone. Leukemia. 2010;24:623-8.

9. Broyl A, Hose D, Lokhorst H, de Knegt Y, Peeters J, Jauch A, et al. Gene expression profiling for molecular classification of multiple myeloma in newly diagnosed patients. Blood. 2010;116: 2543-53.

10. Kumar SK, Uno H, Jacobus SJ, Van Wier SA, Ahmann GJ, Henderson KJ, et al. Impact of gene expression profiling-based risk stratification in patients with myeloma receiving initial therapy with lenalidomide and dexamethasone. Blood 2011 (E-pub ahead of print).

11. Waheed S, Shaughnessy JD, van Rhee F, Alsayed Y, Nair B, Anaissie E, et al. International staging system and metaphase cytogenetic abnormalities in the era of gene expression profiling data in multiple myeloma treated with total therapy 2 and 3 protocols. Cancer. 2011;117:1001-9.

12. Yaccoby $\mathrm{S}$. Advances in the understanding of myeloma bone disease and tumour growth. Br J Haematol. 2010;149:311-21.

13. Fowler JA, Edwards CM, Croucher PI. Tumor-host cell interactions in the bone disease of myeloma. Bone. 2011;48:121-8.

14. Lemaire M, Deleu S, De Bruyne E, Van Valckenborgh E, Menu E, Vanderkerken K. The microenvironment and molecular biology of the multiple myeloma tumor. Adv Cancer Res. 2011;110; $19-42$.

15. Matsumoto T, Abe M. Bone destruction in multiple myeloma. Ann N Y Acad Sci. 2006;1068:319-26.

16. Raje N, Roodman GD. Advances in the biology and treatment of bone disease in multiple myeloma. Clin Cancer Res. 2011;17: $1278-86$.

17. Wu P, Morgan GJ. Targeting Bone as a Therapy for Myeloma. Cancer Microenviron. 2011 (E-pub ahead of print).

18. Hideshima T, Mitsiades C, Tonon G, Richardson PG, Anderson KC. Understanding multiple myeloma pathogenesis in the bone marrow to identify new therapeutic targets. Nat Rev Cancer. 2007;7: 585-98. 